\title{
TỐI ƯU HÓA TIN CẬY VA ĐẬP CỦA ỐNG ĐA TẾ BÀO HìnH VUÔNG CHỊU TẢI VA ĐẬP XIÊN
}

\author{
TRÀ̀N TRỌNG NHÂN \\ Khoa Công nghệ Co khí, Trường Đại học Công nghiệp Thành phố Hồ Chí Minh \\ trantrongnhan@iuh.edu.vn
}

Tóm tắt.Tối ưu hóa tin cậy va đập của những ống hình vuông đa đa tế bào trong trường hợp va đập xiên được nghiên cứu trong bài báo này. Đối với các cấu trúc này, các chỉ số tin cậy va đập SEA và PCF được thu thập bằng cách sử dụng HYPERMESH / LS-DYNA. Pareto front thu được bẳng cách kết hợp response surface (RS) và Non-dominated Sorting Genetic Algorithm II (NSGA-II). Một "giải pháp tốt hơn" (hay còn gọi là knee point) được xác định từ Pareto front. Kết quả của nghiên cứu này là cơ sở tham khảo cho việc thiết kế các cấu trúc đa tế bào có khả năng tin cậy va đập tốt hơn.

Từ khóa: Tin cậy va đập, Ống đa tế bào

\section{OPTIMIZATION OF MULTI-CELL SQUARE COLUMN UNDER OBLIQUE IMPACT}

\begin{abstract}
Crashworthiness optimization of the multi-cell square tubes subjected to oblique impact were studied in this work. For these structures, crashworthiness indicators SEA and PCF are numerically obtained using finite element code HYPERMESH/LS-DYNA. Pareto fronts using a combination of response surface (RS) model and Non-dominated Sorting Genetic Algorithm II (NSGA-II) are obtained. A "better solution" (so-called knee point) from Pareto front with maximizing the reflex angle is determined. The result of this work will assist the design of multi-cell tubes with better crashworthiness performance.
\end{abstract}

Keyword: Crashworthiness, multi-cell column

\section{Lời mở đầu}

Những nghiên cứu về tin cậy va đập dành rất nhiều chú ý đến ứng xử của các cấu trúc thành mỏng, đặc biệt là cấu trúc đa tế bào. Những cấu trúc này được áp dụng như những cấu trúc chịu tải va đập, cũng như hấp thụ năng lượng trong các ứng dụng dành cho ngành ô tô và hàng không. Những quan tâm ngày càng nhiều đối với vấn đề an toàn và phá hủy của các cấu trúc đã dẫn đến những nghiên cứu toàn diện về bài toán va chạm của những cấu trúc thành mỏng với những tiết diện ngang khác nhau [1-5]. Tuy nhiên, một nghiên cứu trước đây về các vụ tai nạn xe trong thực tế cho thấy có tới $90 \%$ cấu trúc thành phần liên quan đến sự phá hủy ở trạng thái uốn. Hơn nữa, người ta đã chỉ ra rằng việc biến dạng theo hướng trục chỉ có thể tái tạo dễ dàng trong các thí nghiệm trong phòng thí nghiệm và các ống có thành mỏng làm việc như những thiết bị hấp thụ năng lượng hiếm khi trải qua tình trạng chịu tải dọc trục thuần túy trong trường hợp va chạm thực sự [6].

Những cấu trúc thành mỏng đa tế bào làm việc như một thành phần hấp thụ năng lượng thường chịu tải va đập xiên. Vào thời điểm đó, các ống phải chịu cả lực dọc trục và mômen uốn. Trong trường hợp ống trải qua sự uốn cong toàn phần, năng lượng hấp thụ của nó sẽ nhỏ hơn so với những trường hợp chịu tải khác [7]. Như thế, cần xem xét tính chất cơ học của các cấu trúc dưới tải va đập xiên. Đồng ý theo mô phỏng, Han và Park [8] đã nghiên cứu trạng thái ứng xử xiên của ống thép vuông. Nghiên cứu của họ cho thấy rằng sự biến dạng lũy tiến dọc trục sẽ được chuyển sang trạng thái uốn toàn phần ở góc tải trọng tới hạn. Reyes và cộng sự. $[9,10]$ đã tiến hành những thí nghiệm trên các ống vuông chịu tải trọng xiên. Nghiên cứu của họ kết luận rằng năng lượng hấp thụ giảm mạnh khi góc tải vượt quá giá trị tới hạn. Qi và cộng sự. [11] đã nghiên cứu trạng thái ứng xử va đập của ống thẳng đơn, ống thành mỏng đa tế bào bằng phương pháp mô phỏng. Công việc của họ cho thấy ống đa tế bào có hiệu suất va đập tốt nhất khi chịu tải xiên. Thêm vào đó, tối ưu hóa đa mục tiêu đối với những cấu trúc trên cũng được thực hiện trong nghiên cứu của Qi và cộng sự. Yang và Qi [12] cũng đã tối ưu hóa tin cậy va đập của các ống thành mỏng hình vuông rỗng và được điền đầy bởi bọt (foam) khi chịu va đập xiên. Song [13] cũng nghiên cứu các ống vuông có cửa sổ chịu va đập xiên bằng phương pháp mô phỏng. Song xem xét ảnh hưởng của góc tải, tham số hình học của 


\section{TỐI UUU HÓA TIN CẬY VA ĐẬP CỦA ỐNG ĐA TẾ BÀO HİNH VUÔNG CHỊU TẢI VA ĐẬP XIÊN}

cửa sổ và tốc độ va đập đến trạng thái ứng xử của cấu trúc. Ngoài ra, tối ưu hóa tin cậy đa mục tiêu (MOD) cũng được áp dụng cho thiết kế tin cậy va đập của các cấu trúc thành mỏng đa tế bào và được thực hiện bởi các nghiên cứu $[4,14,15]$.

Nghiên cứu này tập trung vào ứng xử cũng như tối ưu hóa cấu trúc đa tế bào hình vuông. Đầu tiên, các mô hình phần tử hữu hạn được thiết lập bằng HYPERMESH/ LS-DYNA để xem xét trạng thái ứng xử và đặc tính hấp thụ năng lượng của các cấu trúc đa tế bào. Đồng thời phương pháp đáp ứng bề mặt (Response surface method) được áp dụng để xây dựng quan hệ giữa biến thiết kế và chỉ số tin cậy va đập. Sau đó, thuật toán di truyền NGSA-II được áp dụng để nhận được các giải pháp tối ưu. Cuối cùng, một số kết luận đã được rút ra từ nghiên cứu này.

\section{Mô phỏng (FEA)}

Cấu trúc hình vuông hoặc hình chữ nhật thường được sử dụng trong nhiều cấu trúc va đập. Một trong những lý do là chúng có thể dễ dàng liên kết với những cấu trúc khác. Chính xác hơn, so với các ổng tròn có bề mặt cong, ống vuông hoặc hình chữ nhật có bề mặt phẳng dễ dàng trong việc liên kết với cản trước và các thành phần khác. Trong khi đó, từ phân tích trên, tin cậy va đập của các cấu trúc thành mỏng có thể được tăng cường bằng cách tang cường số góc hoặc áp dụng cấu trúc đa đa tế bào. Từ khái niệm này, cấu trúc hình vuông đa tế bào được đề xuất trong nghiên cứu này (Hình 1 ).

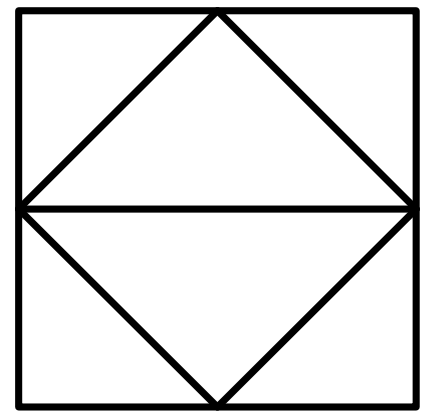

(a): Kiểu I

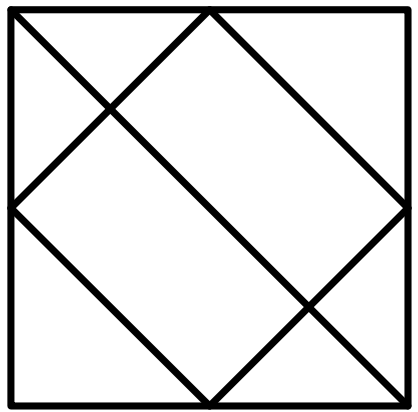

(b): Kiểu II

Hình 1. Cấu trúc hình vuông đa tế bào

Đối với thiết kế tối ưu hóa đa mục tiêu, ảnh hưởng của các tham số hình học (chiều dày ống $\mathrm{t}$ và chiều dài cạnh a) đến khả năng hấp thụ năng lượng được nghiên cứu bằng mô phỏng. Do đó, mô hình $\mathrm{FE}$ đã được thực hiện bằng cách sử dụng HYPERMESH/LS-DYNA để mô phỏng các ống vuông đa tế bào dưới tải trọng tác động xiên. Các cấu trúc là các ống vuông có thành mỏng với 6 và 8 tế bào (xem Hình 1$)$. Chiều dài mặt cắt ngang $\mathrm{a}(80 \mathrm{~mm} \leq \mathrm{a} \leq 100 \mathrm{~mm})$ và độ dày thành ống $\mathrm{t}(1 \mathrm{~mm} \leq \mathrm{t} \leq 2 \mathrm{~mm})$ được định nghĩa là các biến thiết kế. Tất cả các ống dài $250 \mathrm{~mm}$.

Cả hai cấu trúc này được mô hình hóa bằng cách sử dụng các phần tử vỏ bốn nút Belytschko-Tsay với năm điểm tích hợp trong mặt phẳng phần tử. Vật liệu ống AA6060 T4 được mô hình hóa bằng cách sử dụng mô hình vật liệu \# 24 (Mat_Piecewise_Linear_Plasticity) với các tính chất cơ học như: mô đun đàn hồi $\mathrm{E}=$ $68200 \mathrm{MPa}$, ứng suất đàn hồi $\sigma_{\mathrm{y}}=80 \mathrm{MPa}$, ứng suất tới hạn $\sigma_{\mathrm{u}}=173 \mathrm{MP}$, hệ số Poisson $v=0.3$ và hệ số $\mathrm{n}$ $=0.23$ [16]. Vì nhôm không nhạy cảm với tốc độ biến dạng, nên hiệu ứng này bỏ qua trong phân tích phần tử hữu hạn. Kiểu tiếp xúc bề mặt đơn tự động được sử dụng để mô phỏng sự tự tiếp xúc giữa các phần tử vỏ để tránh sự thâm nhập của nếp gấp được tạo ra trong quá trình biến dạng. Đồng thời, kiểu tiếp xúc nút tự động-bề mặt được dùng để mô phỏng tiếp xúc giữa cấu trúc thành mỏng và tường cứng. Hệ số ma sát 0,3 được sử dụng cho tất cả các tương tác tiếp xúc. Để tạo ra đủ động năng, một khối lượng $\mathrm{M}=500 \mathrm{~kg}$ được gắn vào một đầu cấu trúc mẫu thử. Vận tốc va đập là $10 \mathrm{~m} / \mathrm{s}$. Góc tải $\alpha$ là $15^{\circ}$ được sử dụng trong nghiên cứu này. Sơ đồ của mô hình tính toán được thể hiện trong Hình 2. 


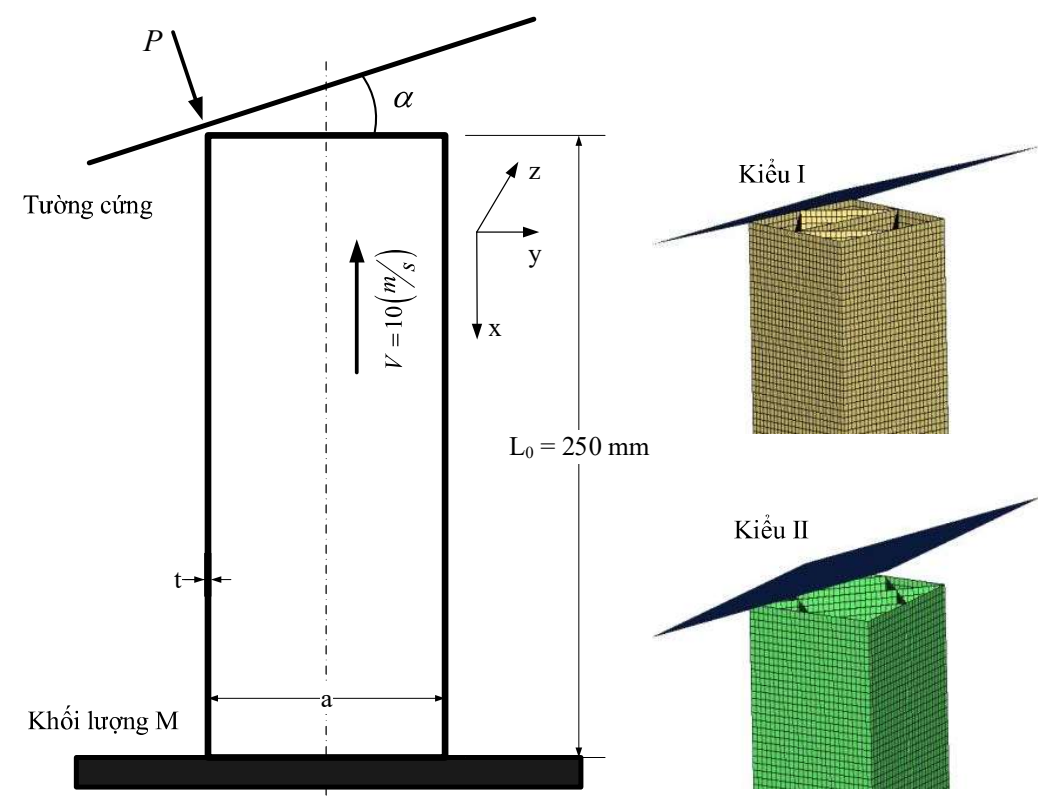

Hình 2. Sơ đồ mô hình tính toán

Như được mô tả trong Hình 1 , tất cả các ống là cấu trúc đa tế bào. Hình 3 cho thấy sự biến dạng của hai cấu trúc. Không có biến dạng uốn cong toàn phần xuất hiện. Hình. 4 cho thấy đồ thị lực - chuyển vị điển hình của Kiểu I và II dưới tải trọng tác động va đập xiên. Lực nghiền, sau khi đạt giá trị lớn nhất, giảm mạnh và sau đó dao động theo chu kỳ xung quanh các giá trị trung bình của lực nghiền tương ứng với sự hình thành biến dạng. Ngoài ra, sự giảm về giá trị của lực nghiền khi chịu va đập không xuất hiện. Điều này có thể giải thích cho việc không có bằng chứng về hiện tượng uốn cong xảy ra khi chịu va đập xiên của những cấu trúc đa tế bào này.

\section{Phương pháp tối ưu hóa đa mục tiêu}

\subsection{Chỉ số tin cậy va đập}

Để định lượng mức độ va đập, một số tiêu chí thường được sử dụng như là lực nghiền cực đại ban đầu (PCF), năng lượng hấp thụ (EA), năng lượng hấp thụ riêng (SEA) [17].

Lực nghiền cực đại ban đầu (PCF) là lực cao nhất trước khi lực nghiền trở nên ổn định. Nó liên quan trực tiếp đển mức độ thương tích của hành khách trong tai nạn. Năng lượng hấp thụ (EA) của cấu trúc được xác định bằng cách tích phân lực tác dụng đối với chuyển vị:

$$
E A=\int_{0}^{l} P(x) d x,
$$

trong đó $\mathrm{P}(\mathrm{x})$ và 1 là lực nghiền dọc trục tức thời tại chuyển vị $\mathrm{x}$ và chuyển vị nghiền đạt được.

Khi đó, năng lượng hấp thụ riêng (SEA) được tính theo công thức:

$$
S E A=\frac{E A}{m},
$$

\subsection{Tối ưu hóa đa mục tiêu dựa trên phương pháp đáp ứng bề mặt}

Đối với các vấn đề va đập-tiếp xúc phi tuyến, rất khó để có được các biểu thức cho các chỉ số tin cậy SEA và $\mathrm{PCF}$. Do đó, mô hình thay thế dựa trên phương pháp đáp ứng bề mặt $(\mathrm{RSM})[4,14,15]$ là một phương pháp hồi quy toán học hiệu quả trong thiết kế tối ưu hóa đa mục tiêu của loại vấn đề này.

Vì lý do an toàn của hành khách, sự giảm tốc càng thấp thì PCF càng nhỏ. Tuy nhiên, sự gia tăng trong SEA thường dẫn đến sự gia tăng PCF. Nói cách khác, SEA có thể tương tác với PCF. Như thế, quá khó để chúng ta có hai mục tiêu được tối ưu hóa đồng thời. Chúng ta phải áp đặt một tối ưu trên Pareto front. Bài toán tối ưu đa mục tiêu của cả hai đối tượng là SEA lớn nhất và $\mathrm{PCF}$ nhỏ nhất sẽ được xác định bằng các phương pháp NSGA-II [18]. Quá trình tối ưu hóa được thể hiện trong Hình 5 cho thấy những mô phỏng 
được thực hiện bởi phần mềm Hypermesh. Kết quả mô phỏng được xử lý bởi phương pháp đáp ứng bề mặt để nhận được hàm mục tiêu. Khi đó phương pháp tối ưu được áp dụng để có kết quả tối ưu.
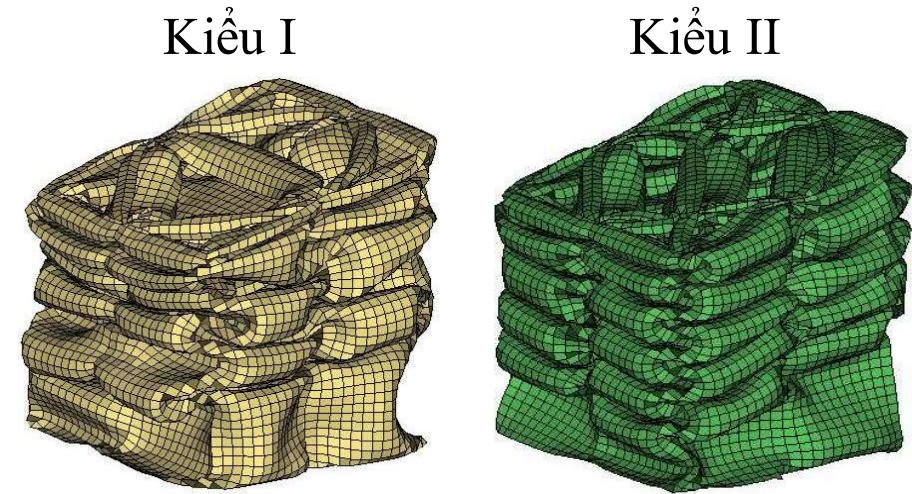

Hình 3. Trạng thái biến dạng của cấu trúc
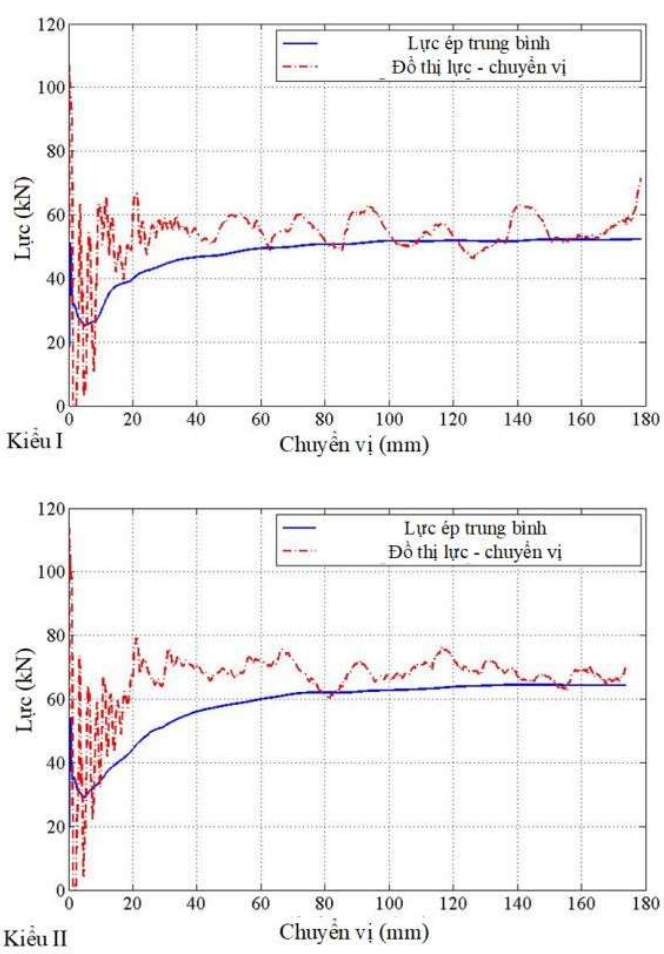

Hình 4. Đồ thị lực - chuyển vị của cấu trúc

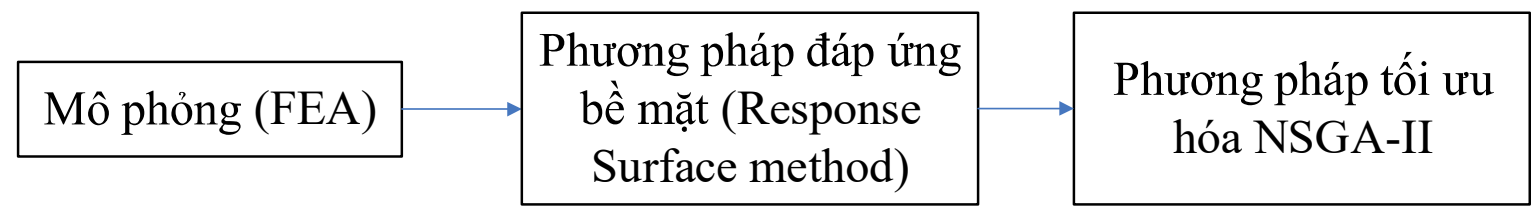

Hình 5. Quá trình tối ưu hóa

Bài toán tối ưu đa mục tiêu có thể được thiết lập công thức như sau 
$\left\{\begin{array}{l}\text { Minimize } \quad(1 / S E A ; P C F) \\ s . t \quad 1 \leq t \leq 2 \mathrm{~mm} \\ \\ \end{array}\right.$

\section{3. Điểm Knee (Knee point)}

Trong nhiều trường hợp, người thiết kế phải có được một giải pháp tốt hơn (được gọi là Knee point) từ các giải pháp tối ưu dựa trên yêu cầu của họ. Có một số phương pháp có thể xác định được Knee point, như là một phương pháp lựa chọn khoảng cách tối thiểu để có được Knee point từ mặt Pareto [19] hoặc thuật toán tiến hóa đa mục tiêu đã sửa đổi [20]. Trong nghiên cứu của chúng tôi, một phương pháp mới đã được phát triển để xác định Knee point bằng cách tối đa hóa góc phản xạ, như trong Hình 6 . Về mặt toán học, công thức được đưa ra là

$$
\left\{\begin{array}{l}
\text { Maximize } \quad \alpha=360^{\circ}-\beta \\
\text { s.t } \quad \cos \beta=\frac{\left|a_{1} a_{2}+b_{1} b_{2}\right|}{\sqrt{a_{1}^{2}+b_{1}^{2}}+\sqrt{a_{2}^{2}+b_{2}^{2}}},
\end{array}\right.
$$

Bảng 1. Phạm vi thiết kế

\begin{tabular}{|c|c|c|c|c|c|c|}
\hline \multirow[b]{2}{*}{ n } & \multirow[b]{2}{*}{ t (mm) } & \multirow[b]{2}{*}{$\mathbf{a}(\mathrm{mm})$} & \multicolumn{2}{|c|}{ Kiểu I } & \multicolumn{2}{|c|}{ kiểu II } \\
\hline & & & $\begin{array}{c}\text { SEA } \\
(\mathbf{k J} / \mathbf{k g})\end{array}$ & $\begin{array}{l}\text { PCF } \\
(\mathbf{k N})\end{array}$ & $\begin{array}{c}\text { SEA } \\
(\mathbf{k J} / \mathbf{k g})\end{array}$ & $\begin{array}{l}\text { PCF } \\
(\mathbf{k N})\end{array}$ \\
\hline 1 & 1 & 80 & 12.914 & 69.331 & 14.135 & 73.385 \\
\hline 2 & 1.25 & 80 & 14.174 & 88.068 & 15.806 & 93.419 \\
\hline 3 & 1.5 & 80 & 15.024 & 107.114 & 17.04 & 113.662 \\
\hline 4 & 1.75 & 80 & 16.169 & 125.74 & 18.355 & 133.311 \\
\hline 5 & 2 & 80 & 17.035 & 143.022 & 19.372 & 151.463 \\
\hline 6 & 1 & 85 & 12.374 & 74.315 & 14.481 & 78.791 \\
\hline 7 & 1.25 & 85 & 14.04 & 94.116 & 15.805 & 99.808 \\
\hline 8 & 1.5 & 85 & 14.814 & 114.467 & 16.928 & 121.127 \\
\hline 9 & 1.75 & 85 & 15.938 & 134.261 & 18.094 & 141.625 \\
\hline 10 & 2 & 85 & 16.416 & 152.533 & 18.371 & 161.44 \\
\hline 11 & 1 & 90 & 11.272 & 78.93 & 13.214 & 83.498 \\
\hline 12 & 1.25 & 90 & 13.27 & 99.949 & 14.392 & 105.921 \\
\hline 13 & 1.5 & 90 & 14.25 & 121.595 & 15.219 & 128.896 \\
\hline 14 & 1.75 & 90 & 14.538 & 142.777 & 16.223 & 151.251 \\
\hline 15 & 2 & 90 & 15.492 & 162.284 & 17.18 & 171.731 \\
\hline 16 & 1 & 95 & 11.003 & 83.773 & 12.638 & 88.596 \\
\hline 17 & 1.25 & 95 & 12.662 & 106.08 & 13.619 & 112.382 \\
\hline 18 & 1.5 & 95 & 13.424 & 129.035 & 14.915 & 136.743 \\
\hline 19 & 1.75 & 95 & 14.238 & 151.289 & 16.008 & 160.211 \\
\hline 20 & 2 & 95 & 14.918 & 171.756 & 16.752 & 181.684 \\
\hline 21 & 1 & 100 & 10.412 & 88.422 & 12.337 & 93.609 \\
\hline 22 & 1.25 & 100 & 11.755 & 111.748 & 13.554 & 118.335 \\
\hline
\end{tabular}




\begin{tabular}{lcccccc}
23 & 1.5 & 100 & 13.003 & 136.175 & 14.821 & 143.939 \\
24 & 1.75 & 100 & 13.519 & 159.917 & 15.649 & 168.569 \\
$\mathbf{2 5}$ & $\mathbf{2}$ & $\mathbf{1 0 0}$ & $\mathbf{1 3 . 6 8 1}$ & $\mathbf{1 8 1 . 9 0 6}$ & $\mathbf{1 6 . 1 1 4}$ & $\mathbf{1 9 1 . 3 0 1}$ \\
\hline
\end{tabular}

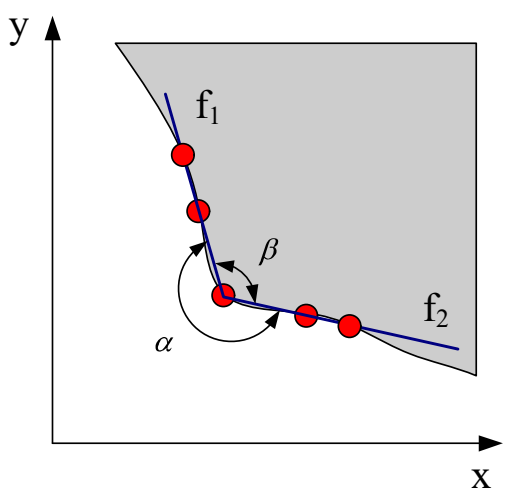

$\left\{\begin{array}{l}f_{1}: a_{1} x+b_{1} y+c_{1}=0 \\ f_{2}: a_{2} x+b_{2} y+c_{2}=0 \\ \alpha=360^{0}-\beta\end{array}\right.$

\section{Tối ưu hóa tin cậy va đập}

Hình 6. Góc phản xạ

Để thiết lập hàm đối tượng đối với SEA và PCF, một loạt 25 điểm mẫu thiết kế đã được chọn trong không gian thiết kế (Bảng 1). Những phân tích về tin cậy va đập được thực hiện trên các hàm đối tượng đó để có được bề mặt đáp ứng (RSM) của SEA và $\mathrm{PCF}$. Theo đó, những bề mặt đáp ứng của $\mathrm{SEA}$ và $\mathrm{PCF}$ cho các ống I và II được vẽ trong Hình 7 . Hình này cho thấy các bề mặt đáp ứng của SEA và PCF ứng xử đơn điệu trên miền thiết kế. SEA và $\mathrm{PCF}$ nghiêng về phía có $\mathrm{a}$ và $\mathrm{t}$ nhỏ. Trong tất cả bề mặt đáp ứng, khoảng sai số tương đối $(\mathrm{RE})$ phù hợp là $(-2.66 \% ; 2.68 \%)$. Khoảng giá trị này được xem là chấp nhận được. Hình 8 cho thấy lỗi tương đối cho tất cả các bề mặt đáp ứng tại tất cả những điểm lấy mẫu thiết kế.
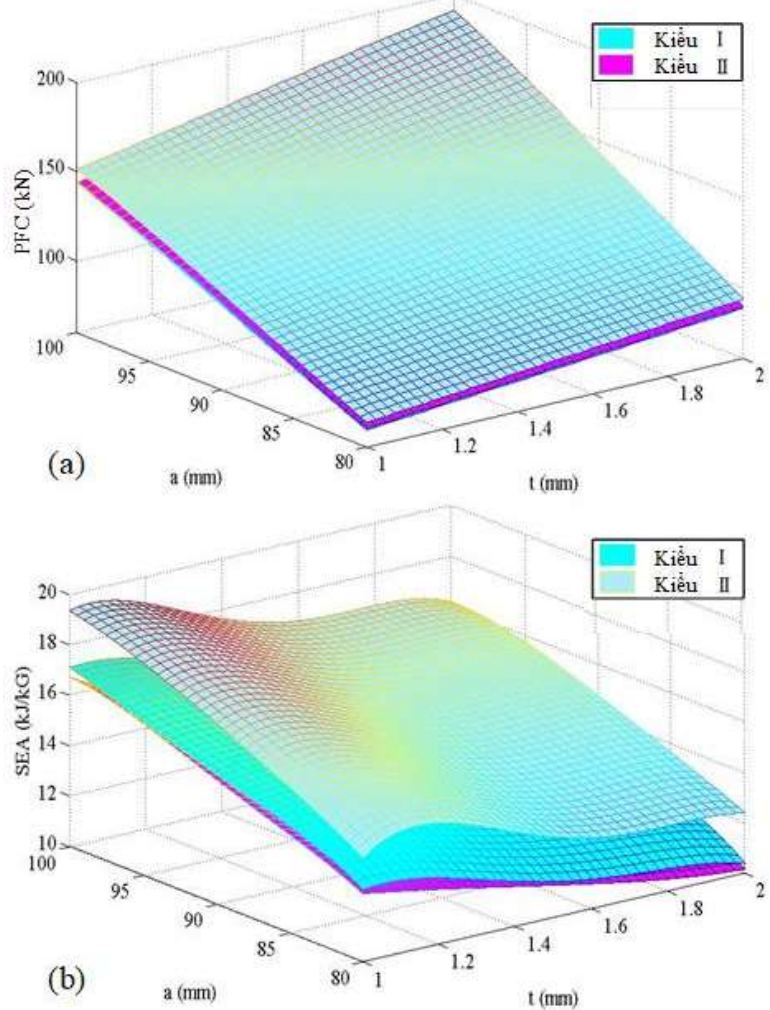

Hình 7. Bề mặt đáp ứng của Kiểu I và II 

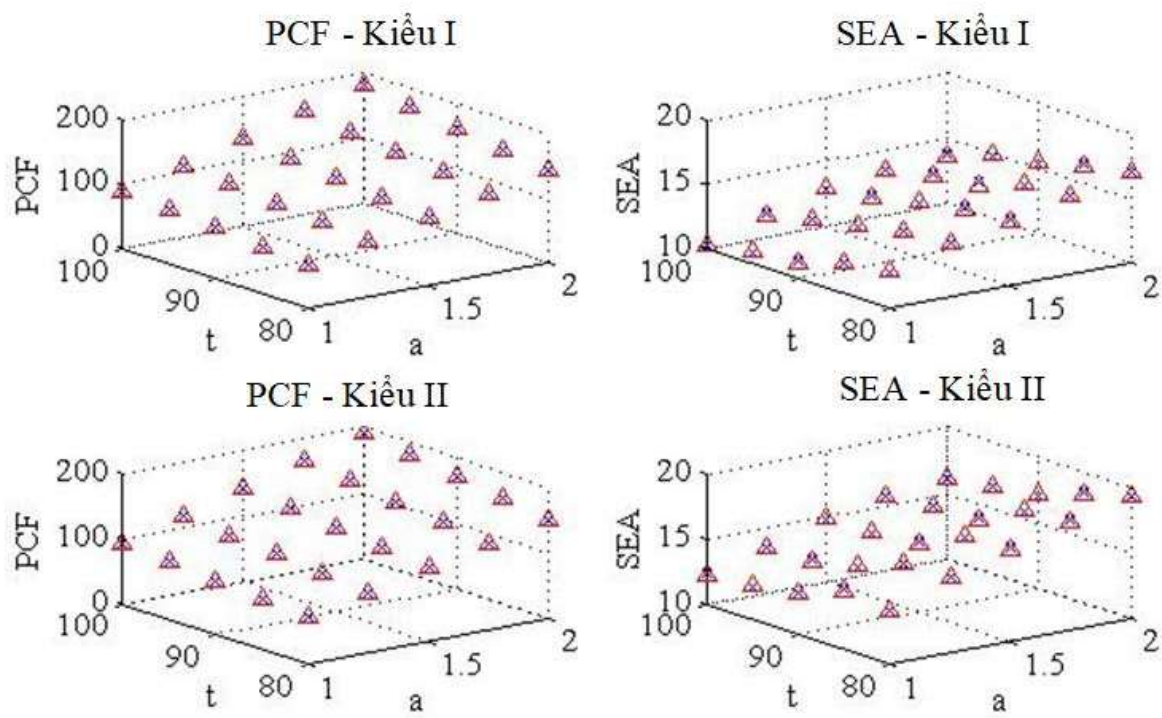

* Kết quả mô phỏng $\Delta$ Kết quả RSM

Hình 8. Khoảng sai số tương đối (RE)

Các mặt Pareto của 1/SEA so với PCF cho kiểu cấu trúc được vẽ trong Hình 9. Đồng thời, các mặt Pareto hiển thị các điểm giải pháp phân bố đồng đều trong không gian Pareto. Trên thực tế, bất kỳ điểm nào trên biên giới Pareto đều có thể là một kết quả tối ưu và một loạt các giải pháp tối ưu được cung cấp cho người ra quyết định. Đó là lý do tại sao một số giải pháp được đề xuất để phát hiện giải pháp tốt hơn (Knee point). Theo đó, một phương pháp xác định Knee point với tối đa hóa góc phản xạ $\alpha$ đã được đề xuất như trong Hình 6. Kết quả của phương trình. (4) cho thấy các điểm đầu gối với góc phản xạ tối đa hóa đối với loại ống I và II lần lượt là $183.25^{\circ}$ và $185.7^{\circ}$ như trong Hình 9 . Knee point của Kiểu I và II trong trường hợp va đập xiên được trình bày trong Bảng 2 . Các sai số tương đối RE giữa kết quả mô phỏng và dự đoán RSM cũng được tóm tắt trong Bảng 2 . Sai số RE cho thấy RSM có độ chính xác cao.
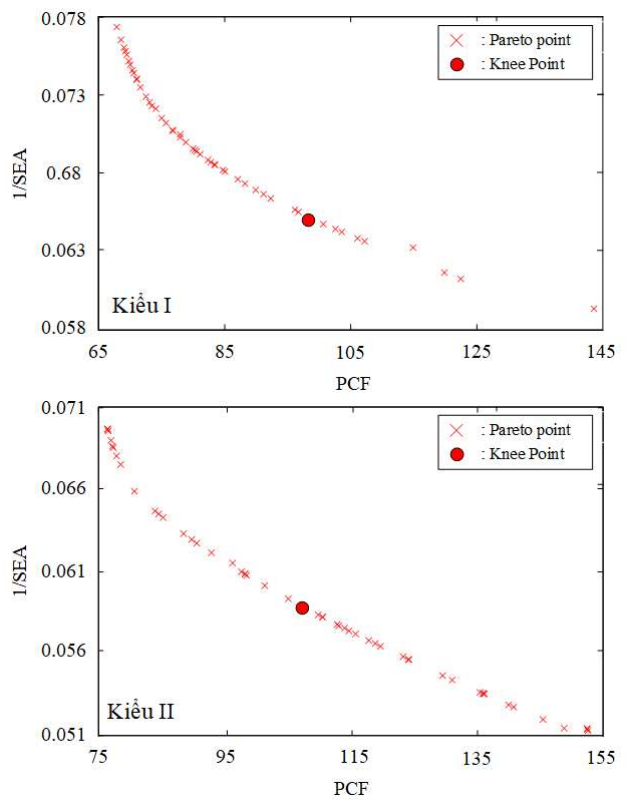

Hình 9. Mặt Pareto 


\section{TỐI UUU HÓA TIN CẬY VA ĐẬP CỦA ỐNG ĐA TẾ BÀO HİNH VUÔNG CHİU TẢI VA ĐẬP XIÊN}

Bảng 2. Kết quả tối ưu

\begin{tabular}{|c|c|c|c|c|}
\hline Kiểu & Thuật ngũ̃ & $\begin{array}{c}\text { Giá trị tối ưu } \\
(\mathbf{m m})\end{array}$ & $\operatorname{SEA}(\mathbf{k J} / \mathbf{k N})$ & $\operatorname{PCF}(k N)$ \\
\hline \multirow{3}{*}{ Kiểu I } & Giá trị xấp xỉ & & 15.165 & 99.023 \\
\hline & $\begin{array}{l}\text { Kết quả của } \\
\text { FEA }\end{array}$ & $t=1.421, a=80.056$ & 15.347 & 98.885 \\
\hline & RE (\%) & & -1.177 & 0.139 \\
\hline \multirow{3}{*}{ Kiểu II } & Giá trị xấp xỉ & & 17.246 & 108.157 \\
\hline & $\begin{array}{c}\text { Kết quả của } \\
\text { FEA }\end{array}$ & $t=1.443, a=80.875$ & 17.548 & 107.996 \\
\hline & RE (\%) & & -1.271 & 0.149 \\
\hline
\end{tabular}

\section{Kết luận}

Trong nghiên cứu này, tối ưu hóa tin cậy va đập của hai kiểu ống đa tế bào đã được đề xuất và nghiên cứu. Thiết kế tối ưu hóa của các ống này được thực hiện bằng kết hợp FEA, RSM và NSGA-II. Bề mặt đáp ứng của $\mathrm{PCF}$ và $\mathrm{SEA}$ được thiết lập cho hai kiểu cấu trúc I và II. Bề mặt Pareto thu được bằng phương pháp NSGA-II. Một phương pháp mới đã được giới thiệu để tìm ra giải pháp tốt hơn (Knee point) từ mặt Pareto. Sai số tương đối giữa giá trị dự đoán RSM và kết quả mô phỏng FEA là chấp nhận được. Kết quả của nghiên cứu này sẽ hỗ trợ cho việc thiết kế các cấu trúc đa tế bào ứng dụng trong bộ phận hấp thụ năng lương va đập.

\section{Tham khảo}

[1] S. Hou, Q. Li, S. Long, X. Yang, and W. Li, "Crashworthiness design for foam filled thin-wall structures," Materials \& Design, vol. 30, no. 6, pp. 2024-2032, 6// 2009.

[2] Y. Zhang, G. Sun, G. Li, Z. Luo, and Q. Li, "Optimization of foam-filled bitubal structures for crashworthiness criteria," Materials \& Design, vol. 38, no. 0, pp. 99-109, 6// 2012.

[3] H. Yin, G. Wen, S. Hou, and K. Chen, "Crushing analysis and multiobjective crashworthiness optimization of honeycomb-filled single and bitubular polygonal tubes," Materials \& Design, vol. 32, no. 8-9, pp. 44494460, 9// 2011.

[4] S. Hou, Q. Li, S. Long, X. Yang, and W. Li, "Multiobjective optimization of multi-cell sections for the crashworthiness design," International Journal of Impact Engineering, vol. 35, no. 11, pp. 1355-1367, 11// 2008.

[5] G. H. Daneshi and S. J. Hosseinipour, "Grooves effect on crashworthiness characteristics of thin-walled tubes under axial compression," Materials \& Design, vol. 23, no. 7, pp. 611-617, 10// 2002.

[6] W. Chen, "Experimental and numerical study on bending collapse of aluminum foam-filled hat profiles," International Journal of Solids and Structures, vol. 38, no. 44, pp. 7919-7944, 2001/11/01/ 2001.

[7] F. Tarlochan, F. Samer, A. M. S. Hamouda, S. Ramesh, and K. Khalid, "Design of thin wall structures for energy absorption applications: Enhancement of crashworthiness due to axial and oblique impact forces," Thin-Walled Structures, vol. 71, no. 0, pp. 7-17, 10// 2013.

[8] D. C. Han and S. H. Park, "Collapse behavior of square thin-walled columns subjected to oblique loads," Thin-Walled Structures, vol. 35, no. 3, pp. 167-184, 11// 1999.

[9] A. Reyes, M. Langseth, and O. S. Hopperstad, "Crashworthiness of aluminum extrusions subjected to oblique loading: experiments and numerical analyses," International Journal of Mechanical Sciences, vol. 44, no. 9 , pp. 1965-1984, 9// 2002.

[10] A. Reyes, M. Langseth, and O. S. Hopperstad, "Square aluminum tubes subjected to oblique loading," International Journal of Impact Engineering, vol. 28, no. 10, pp. 1077-1106, 11// 2003.

[11] C. Qi, S. Yang, and F. Dong, "Crushing analysis and multiobjective crashworthiness optimization of tapered square tubes under oblique impact loading," Thin-Walled Structures, vol. 59, no. 0, pp. 103-119, 10// 2012. 

CHỊU TẢI VA ĐẬP XIÊN

[12] S. Yang and C. Qi, "Multiobjective optimization for empty and foam-filled square columns under oblique impact loading," International Journal of Impact Engineering, vol. 54, no. 0, pp. 177-191, 4// 2013.

[13] J. Song, "Numerical simulation on windowed tubes subjected to oblique impact loading and a new method for the design of obliquely loaded tubes," International Journal of Impact Engineering, vol. 54, no. 0, pp. 192-205, 4// 2013.

[14] S. Chen, H. Yu, and J. Fang, "A novel multi-cell tubal structure with circular corners for crashworthiness," Thin-Walled Structures, vol. 122, pp. 329-343, 2018/01/01/ 2018.

[15] L. Zhang, Z. Bai, and F. Bai, "Crashworthiness design for bio-inspired multi-cell tubes with quadrilateral, hexagonal and octagonal sections," Thin-Walled Structures, vol. 122, pp. 42-51, 2018/01/01/ 2018.

[16] S. P. Santosa, T. Wierzbicki, A. G. Hanssen, and M. Langseth, "Experimental and numerical studies of foamfilled sections," International Journal of Impact Engineering, vol. 24, no. 5, pp. 509-534, 5// 2000.

[17] A. G. Hanssen, M. Langseth, and O. S. Hopperstad, "Static and dynamic crushing of circular aluminium extrusions with aluminium foam filler," International Journal of Impact Engineering, vol. 24, no. 5, pp. 475$507,5 / / 2000$.

[18] F. Usta, Z. Eren, H. Kurtaran, H. S. Türkmen, Z. Kazanc1, and Z. Mecitoglu, "Crashworthiness Optimization of Nested and Concentric Circular Tubes Using Response Surface Methodology and Genetic Algorithm \%J Latin American Journal of Solids and Structures," vol. 15, 2018.

[19] G. Sun, G. Li, S. Zhou, H. Li, S. Hou, and Q. Li, "Crashworthiness design of vehicle by using multiobjective robust optimization," (in English), Structural and Multidisciplinary Optimization, vol. 44, no. 1, pp. 99-110, 2011/07/01 2011.

[20] J. Branke, K. Deb, H. Dierolf, and M. Osswald, "Finding Knees in Multi-objective Optimization," in Parallel Problem Solving from Nature - PPSN VIII, vol. 3242, X. Yao et al., Eds. (Lecture Notes in Computer Science: Springer Berlin Heidelberg, 2004, pp. 722-731.

Ngày nhận bài: 09/10/2019

Ngày chấp nhận đăng:01/04/2020 